\title{
Note on the Stability Property of a Cooperative System Incorporating Harvesting
}

\author{
Xiangdong Xie, ${ }^{1}$ Fengde Chen, ${ }^{1}$ and Yalong $\mathrm{Xue}^{2}$ \\ ${ }^{1}$ Department of Mathematics, Ningde Normal University, Ningde, Fujian 352100, China \\ ${ }^{2}$ College of Mathematics and Computer Science, Fuzhou University, Fuzhou, Fujian 350108, China
}

Correspondence should be addressed to Xiangdong Xie; ndsyxxd@163.com

Received 13 January 2014; Accepted 9 March 2014; Published 2 April 2014

Academic Editor: Jagannathan Sarangapani

Copyright (c) 2014 Xiangdong Xie et al. This is an open access article distributed under the Creative Commons Attribution License, which permits unrestricted use, distribution, and reproduction in any medium, provided the original work is properly cited.

The stability of a kind of cooperative model incorporating harvesting is revisited in this paper. By using an iterative method, the global attractivity of the interior equilibrium point of the system is investigated. We show that the condition which ensures the existence of a unique positive equilibrium is enough to ensure the global attractivity of the positive equilibrium. Our results significantly improve the corresponding results of Wei and Li (2013).

\section{Introduction}

In [1], Wei and Li proposed and studied the following cooperative system incorporating harvesting:

$$
\begin{aligned}
& \dot{x}=x\left(r_{1}-b_{1} x-\frac{a_{1} x}{y+k_{1}}\right)-E q x, \\
& \dot{y}=y\left(r_{2}-b_{2} y-\frac{a_{2} y}{x+k_{2}}\right),
\end{aligned}
$$

where $x$ and $y$ denote the densities of two populations at time $t$. The parameters $r_{1}, r_{2}, a_{1}, a_{2}, b_{1}, b_{2}, k_{1}, k_{2}, E, q$ are all positive constants. Assume that $r_{1}>E q$; then, the equilibria of (1) are

$$
\begin{gathered}
H_{0}(0,0), \quad H_{1}\left(0, \frac{r_{2} k_{2}}{a_{2}+k_{2} b}\right), \\
H_{3}\left(\frac{\left(r_{1}-E q\right) k_{1}}{a_{1}+k_{1} b_{1}}, 0\right),
\end{gathered}
$$

where

$$
\begin{aligned}
x^{*} & =\frac{-\left(k_{2} P-F\right)+\sqrt{\left(k_{2} P-F\right)^{2}+4 P Q M}}{2 P}, \\
y^{*} & =\frac{r_{2}\left(x^{*}+k_{2}\right)}{b_{2} x^{*}+a_{2}+k_{2} b_{2}}, \quad P=r_{2} b_{1}+k_{1} b_{1} b_{2}+a_{1} b_{2}, \\
Q & =r_{1}-E q, \quad F=r_{2} Q+b_{2} k_{1} Q-k_{1} a_{2} b_{1}-a_{1} a_{2}, \\
M & =r_{2} k_{2}+k_{1} k_{2} b_{2}+a_{2} k_{1} .
\end{aligned}
$$

Wei and Li had showed that $H_{0}, H_{1}, H_{2}$ are unstable and concerned with the persistence and stability property of the system; by applying the comparison theorem of differential equations and constructing a suitable Lyapunov function, they obtained the following results.

Theorem A. If $r_{1}>E q, k_{1} b_{1}>a_{1}, k_{2} b_{2}>a_{2}$, then the system (1) is persistent. More precisely,

$$
\begin{aligned}
& C \leq \liminf _{t \rightarrow+\infty} x(t) \leq \limsup _{t \rightarrow+\infty} x(t) \leq A, \\
& D \leq \liminf _{t \rightarrow+\infty} x(t) \leq \limsup _{t \rightarrow+\infty} y(t) \leq B,
\end{aligned}
$$




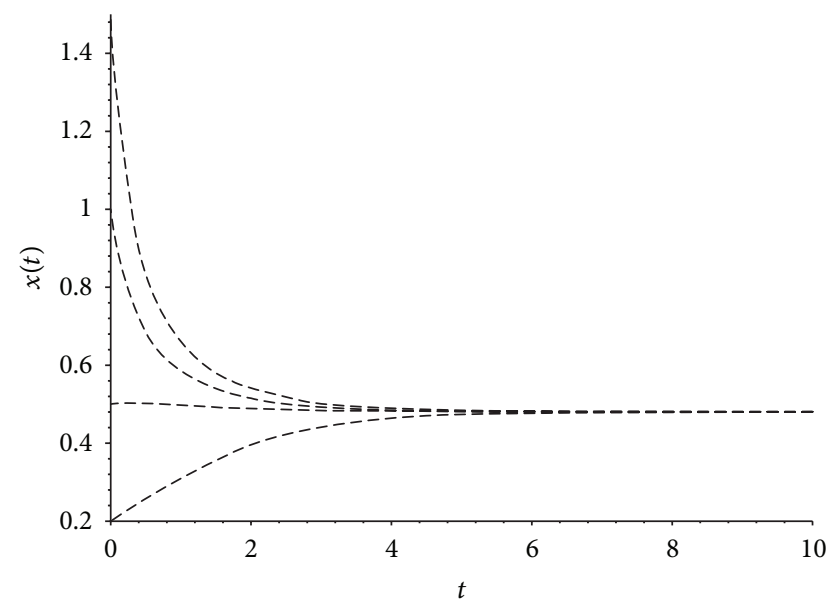

(a)

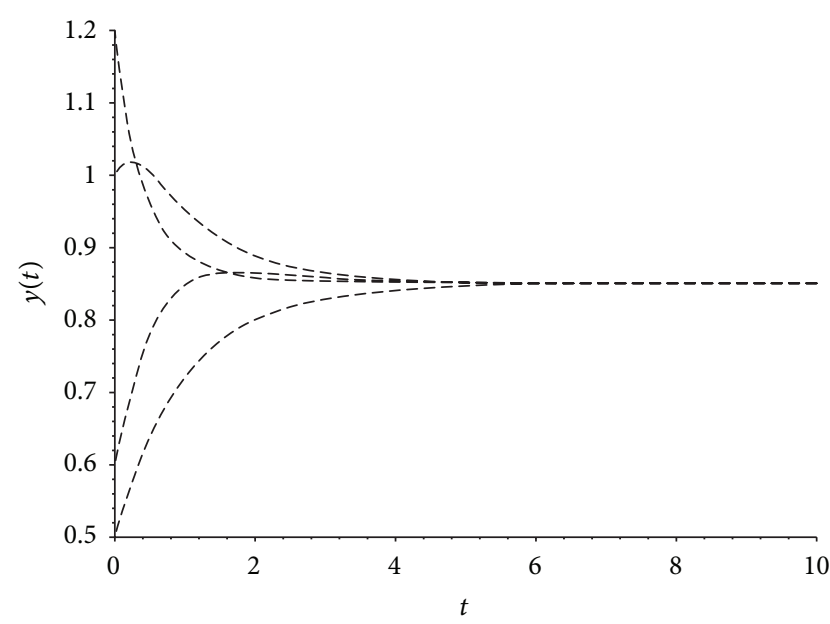

(b)

FIGURE 1: Dynamics behaviors of system (7). Here, we take the initial conditions $\left(x_{1}(0), x_{2}(0)\right)=(0.5,1.2),(1.5,1),(0.2,0.5)$ and $(1,0.6)$, respectively.

where

$$
\begin{array}{ll}
A=\frac{r_{1}-E q}{b_{1}}, \quad B=\frac{r_{2}}{b_{2}}, & \\
C=\frac{\left(r_{1}-E q\right)\left(k_{1} b_{1}-a_{1}\right)}{b_{1}^{2} k_{1}}, \quad D=\frac{r_{2}\left(k_{2} b_{2}-a_{2}\right)}{b_{2}^{2} k_{2}} .
\end{array}
$$

Theorem B. If $r_{1}>E q, k_{1} b_{1}>a_{1}, k_{2} b_{2}>a_{2}$,

$$
\begin{aligned}
& b_{1}+\frac{a_{1}}{y^{*}+k_{1}}>\frac{B a_{2}}{2\left(C+k_{2}\right)\left(x^{*}+k_{2}\right)}+\frac{A a_{1}}{2\left(D+k_{1}\right)\left(y^{*}+k_{1}\right)}, \\
& b_{2}+\frac{a_{2}}{x^{*}+k_{2}}>\frac{B a_{2}}{2\left(C+k_{2}\right)\left(x^{*}+k_{2}\right)}+\frac{A a_{1}}{2\left(D+k_{1}\right)\left(y^{*}+k_{1}\right)},
\end{aligned}
$$

where $A, B, C, D$ are defined by Theorem $A$, then the positive equilibrium point $\mathrm{H}_{3}$ of system (1) is globally asymptotically stable.

Now let us consider the following example.

Example 1. We have

$$
\begin{aligned}
& \dot{x}=x\left(2-x-\frac{2 x}{y+1}\right)-x, \\
& \dot{y}=y\left(2-y-\frac{2 y}{x+1}\right) .
\end{aligned}
$$

Here we choose $r_{1}=r_{2}=2, b_{1}=b_{2}=1, k_{1}=$ $k_{2}=1, a_{1}=a_{2}=2, E=q=1$, and the parameters $r_{1}, r_{2}, a_{1}, a_{2}, b_{1}, b_{2}, k_{1}, k_{2}, E, q$ are all positive constants. Obviously, $r_{1}=2>1=E q, k_{1} b_{1}=1<2=a_{1}, k_{2} b_{2}=$ $1<2=a_{2}$. Hence, the conditions of Theorems $\mathrm{A}$ and $\mathrm{B}$ are not all satisfied; however, numeric simulations (Figure 1) show that the unique positive equilibrium $(0.4806248475$, 0.8507810594 ) is globally attractive.
The above example shows that it is possible to obtain some weaker conditions than those of Theorems A and B to ensure the persistent and stability of the system. The aim of this paper is to prove the following result.

Theorem 2. Assume that $r_{1}>$ Eq holds; then, the unique positive equilibrium $E^{*}\left(x^{*}, y^{*}\right)$ is globally attractive; that is,

$$
\lim _{t \rightarrow+\infty} x(t)=x^{*}, \quad \lim _{t \rightarrow+\infty} y(t)=y^{*}
$$

Concerned with the persistent property of the system, as a direct corollary of Theorem 2, we have the following.

Corollary 3. Assume that $r_{1}>$ Eq holds; then, system (1) is permanent.

Remark 4. A comparison of Theorems A, B, and 2 and Corollary 3 shows that $k_{1} b_{1}>a_{1}, k_{2} a_{2}>b_{2}$, and inequalities (6) are redundant. Therefore, our results significantly improve the corresponding main results of Wei and $\mathrm{Li}[1]$.

We will prove Theorem 2 in the next section. For more works on mutualism system, one could refer to [2-10] and the references cited therein.

\section{Proof of the Main Results}

As a direct corollary of Lemma 2.2 of Chen [11], we have the following.

Lemma 5. If $a>0, b>0$ and $\dot{x} \geq x(b-a x)$, when $t \geq 0$ and $x(0)>0$, we have

$$
\liminf _{t \rightarrow+\infty} x(t) \geq \frac{b}{a}
$$


If $a>0, b>0$ and $\dot{x} \leq x(b-a x)$, when $t \geq 0$ and $x(0)>0$, we have

$$
\limsup _{t \rightarrow+\infty} x(t) \leq \frac{b}{a} .
$$

Proof of Theorem 2. By the first equation of system (1), we have

$$
\dot{x}(t) \leq x(t)\left(r_{1}-E q-b_{1} x(t)\right) .
$$

From Lemma 5, it follows that

$$
\limsup _{t \rightarrow+\infty} x(t) \leq \frac{r_{1}-E q}{b_{1}} .
$$

Hence, for enough small $\varepsilon>0\left(\varepsilon<\min \left\{\left(r_{1}-E q\right) k_{1} /\left(k_{1} b_{1}+\right.\right.\right.$ $\left.\left.\left.a_{1}\right), r_{2} k_{2} /\left(k_{2} b_{2}+a_{2}\right)\right\}\right)$, it follows from (12) that there exists a $T_{1}^{\prime}>0$ such that

$$
x(t)<\frac{r_{1}-E q}{b_{1}}+\varepsilon \stackrel{\text { def }}{=} M_{1}^{(1)} \quad \forall t>T_{1}^{\prime} .
$$

Similarly, for the above $\varepsilon>0$, it follows from the second equation of system (1) that there exists a $T_{1}>T_{1}^{\prime}$ such that

$$
y(t)<\frac{r_{2}}{b_{2}}+\varepsilon \stackrel{\text { def }}{=} M_{2}^{(1)} \quad \forall t>T_{1} .
$$

(14) together with the first equation of system (1) implies that

$$
\begin{aligned}
\dot{x} & =x\left(r_{1}-b_{1} x-\frac{a_{1} x}{y+k_{1}}\right)-E q x \\
& \leq x\left(r_{1}-E q-b_{1} x-\frac{a_{1} x}{M_{2}^{(1)}+k_{1}}\right) \quad \forall t>T_{1} .
\end{aligned}
$$

Therefore, by Lemma 5, we have

$$
\limsup _{t \rightarrow+\infty} x_{1}(t) \leq \frac{r_{1}-E q}{b_{1}+\left(a_{1} /\left(M_{2}^{(1)}+k_{1}\right)\right)} .
$$

That is, for $\varepsilon>0$ to be defined by (12) and (13), there exists a $T_{2}^{\prime}>T_{1}$ such that

$$
x(t)<\frac{r_{1}-E q}{b_{1}+\left(a_{1} /\left(M_{2}^{(1)}+k_{1}\right)\right)}+\frac{\varepsilon}{2} \stackrel{\text { def }}{=} M_{1}^{(2)}>0 \quad \forall t>T_{2}^{\prime} .
$$

It follows from (13) and the second equation of system (1) that

$$
\begin{aligned}
\dot{y} & =y\left(r_{2}-b_{2} y-\frac{a_{2} y}{x+k_{2}}\right) \\
& \leq y\left(r_{2}-b_{2} y-\frac{a_{2} y}{M_{1}^{(1)}+k_{2}}\right) .
\end{aligned}
$$

Therefore, by Lemma 5, we have

$$
\limsup _{t \rightarrow+\infty} y(t) \leq \frac{r_{2}}{b_{2}+\left(a_{2} /\left(M_{1}^{(1)}+k_{2}\right)\right)} .
$$

That is, for $\varepsilon>0$ to be defined by (13) and (14), there exists a $T_{2}>T_{2}^{\prime}$ such that

$$
y(t)<\frac{r_{2}}{b_{2}+\left(a_{2} /\left(M_{1}^{(1)}+k_{2}\right)\right)}+\frac{\varepsilon}{2} \stackrel{\text { def }}{=} M_{2}^{(2)}>0 \quad \forall t>T_{2} .
$$

From the first equation of system (1) and the positivity of $y(t)$, we have

$$
\begin{aligned}
\dot{x} & =x\left(r_{1}-b_{1} x-\frac{a_{1} x}{y+k_{1}}\right)-E q x \\
& \geq x\left(r_{1}-E q-b_{1} x-\frac{a_{1} x}{k_{1}}\right) \quad \forall t>T_{2} .
\end{aligned}
$$

Therefore, by Lemma 5, we have

$$
\liminf _{t \rightarrow+\infty} x(t) \geq \frac{r_{1}-E q}{b_{1}+\left(a_{1} / k_{1}\right)} .
$$

Hence, for $\varepsilon>0$ to be defined by (12) and (13), there exists a $T_{3}^{\prime}>T_{2}$ such that

$$
x(t)>\frac{r_{1}-E q}{b_{1}+\left(a_{1} / k_{1}\right)}-\varepsilon \stackrel{\text { def }}{=} m_{1}^{(1)}, \quad \forall t>T_{3}^{\prime} .
$$

Similarly, it follows from the second equation of system (1) that there exists a $T_{3}>T_{3}^{\prime}$ such that

$$
y(t)>\frac{r_{2}}{b_{2}+\left(a_{2} / k_{2}\right)}-\varepsilon \stackrel{\text { def }}{=} m_{2}^{(1)}, \quad \forall t>T_{3} .
$$

(24) together with the first equation of system (1) implies that

$$
\begin{aligned}
\dot{x} & =x\left(r_{1}-b_{1} x-\frac{a_{1} x}{y+k_{1}}\right)-E q x \\
& \geq x\left(r_{1}-E q-b_{1} x-\frac{a_{1} x}{m_{2}^{(1)}+k_{1}}\right) \quad \forall t>T_{3} .
\end{aligned}
$$

Therefore, by Lemma 5, we have

$$
\liminf _{t \rightarrow+\infty} x(t) \geq \frac{r_{1}-E q}{b_{1}+\left(a_{1} /\left(m_{2}^{(1)}+k_{1}\right)\right)} .
$$

That is, for $\varepsilon>0$ to be defined by (12) and (13), there exists a $T_{4}^{\prime}>T_{3}$ such that

$$
x(t)>\frac{r_{1}-E q}{b_{1}+\left(a_{1} /\left(m_{2}^{(1)}+k_{1}\right)\right)}-\frac{\varepsilon}{2} \stackrel{\text { def }}{=} m_{1}^{(2)}>0, \quad \forall t>T_{4}^{\prime} .
$$

Similarly, by (23) and the second equation of system (1), for $\varepsilon>0$ to be defined by (12) and (13), there exists a $T_{4}>T_{4}^{\prime}$ such that

$$
y(t)>\frac{r_{2}}{b_{2}+\left(a_{2} /\left(m_{1}^{(1)}+k_{2}\right)\right)}-\frac{\varepsilon}{2} \stackrel{\text { def }}{=} m_{2}^{(2)}>0, \quad \forall t>T_{4} .
$$


Noting that $a_{1} /\left(M_{2}^{(1)}+k_{1}\right)>0, a_{2} /\left(M_{1}^{(1)}+k_{2}\right)>0$, it immediately follows that

$$
\begin{aligned}
& M_{1}^{(2)}=\frac{r_{1}-E q}{b_{1}+\left(a_{1} /\left(M_{2}^{(1)}+k_{1}\right)\right)}+\frac{\varepsilon}{2}<\frac{r_{1}-E q}{b_{1}}+\varepsilon=M_{1}^{(1)} ; \\
& M_{2}^{(2)}=\frac{r_{2}}{b_{2}+\left(a_{2} /\left(M_{1}^{(1)}+k_{2}\right)\right)}+\frac{\varepsilon}{2}<\frac{r_{2}}{b_{2}}+\varepsilon=M_{2}^{(1)} .
\end{aligned}
$$

Also, since $m_{1}^{(1)}>0, m_{2}^{(1)}>0$, it follows that $a_{1} /\left(m_{2}^{(1)}+k_{1}\right)<$ $a_{1} / k_{1}, a_{2} /\left(m_{1}^{(1)}+k_{2}\right)<a_{2} / k_{2}$, and so

$m_{1}^{(2)}=\frac{r_{1}-E q}{b_{1}+\left(a_{1} /\left(m_{2}^{(1)}+k_{1}\right)\right)}-\frac{\varepsilon}{2}>\frac{r_{1}-E q}{b_{1}+\left(a_{1} / k_{1}\right)}-\varepsilon=m_{1}^{(1)} ;$

$m_{2}^{(2)}=\frac{r_{2}}{b_{2}+\left(a_{2} /\left(m_{1}^{(1)}+k_{2}\right)\right)}-\frac{\varepsilon}{2}>\frac{r_{2}}{b_{2}+\left(a_{2} / k_{2}\right)}-\varepsilon=m_{2}^{(1)}$.

Repeating the above procedure, we get four sequences $M_{i}^{(n)}$, $m_{i}^{(n)}, i=1,2, n=1,2, \ldots$, such that for $n \geq 2$

$$
\begin{aligned}
& M_{1}^{(n)}=\frac{r_{1}-E q}{b_{1}+\left(a_{1} /\left(M_{2}^{(n-1)}+k_{1}\right)\right)}+\frac{\varepsilon}{n} ; \\
& M_{2}^{(n)}=\frac{r_{2}}{b_{2}+\left(a_{2} /\left(M_{1}^{(n-1)}+k_{2}\right)\right)}+\frac{\varepsilon}{n} ; \\
& m_{1}^{(n)}=\frac{r_{1}-E q}{b_{1}+\left(a_{1} /\left(m_{2}^{(n-1)}+k_{1}\right)\right)}-\frac{\varepsilon}{n} ; \\
& m_{2}^{(n)}=\frac{r_{2}}{b_{2}+\left(a_{2} /\left(m_{1}^{(n-1)}+k_{2}\right)\right)}-\frac{\varepsilon}{n} .
\end{aligned}
$$

Obviously,

$$
m_{i}^{(n)}<x_{i}(t)<M_{i}^{(n)} \quad \forall t \geq T_{2 n}, i=1,2 .
$$

We claim that sequences $M_{i}^{(n)}, i=1,2$ are strictly decreasing, and sequences $m_{i}^{(n)}, i=1,2$ are strictly increasing. To proof this claim, we will carry them out by induction. Firstly, from (29) and (30) we have

$$
M_{i}^{(2)}<M_{i}^{(1)}, \quad m_{i}^{(2)}>m_{i}^{(1)}, \quad i=1,2 .
$$

Let us assume now that our claim is true for $n$; that is,

$$
M_{i}^{(n)}<M_{i}^{(n-1)}, \quad m_{i}^{(n)}>m_{i}^{(n-1)}, \quad i=1,2 .
$$

Then,

$$
\begin{gathered}
\frac{a_{1}}{M_{2}^{(n)}+k_{1}}>\frac{a_{1}}{M_{2}^{(n-1)}+k_{1}}, \\
\frac{r_{2}}{b_{2}+\left(a_{2} /\left(M_{1}^{(n)}+k_{2}\right)\right)}>\frac{r_{2}}{b_{2}+\left(a_{2} /\left(M_{1}^{(n-1)}+k_{2}\right)\right)} .
\end{gathered}
$$

From (34) and the expression of $M_{i}^{(n)}$, it immediately follows that

$$
\begin{aligned}
M_{1}^{(n+1)} & =\frac{r_{1}-E q}{b_{1}+\left(a_{1} /\left(M_{2}^{(n)}+k_{1}\right)\right)}+\frac{\varepsilon}{n+1} \\
& <\frac{r_{1}-E q}{b_{1}+\left(a_{1} /\left(M_{2}^{(n-1)}+k_{1}\right)\right)}+\frac{\varepsilon}{n}=M_{1}^{(n)}, \\
M_{2}^{(n+1)} & =\frac{r_{2}}{b_{2}+\left(a_{2} /\left(M_{1}^{(n)}+k_{2}\right)\right)}+\frac{\varepsilon}{n+1} \\
& <\frac{r_{2}}{b_{2}+\left(a_{2} /\left(M_{1}^{(n-1)}+k_{2}\right)\right)}+\frac{\varepsilon}{n}=M_{2}^{(n)} .
\end{aligned}
$$

Also, it follows from (34) that $m_{i}^{(n)} \geq m_{i}^{(n-1)}, i=1,2$. Then,

$$
\frac{a_{1}}{m_{2}^{(n)}+k_{1}}<\frac{a_{1}}{m_{2}^{(n-1)}+k_{1}}, \quad \frac{a_{2}}{m_{1}^{(n)}+k_{2}}<\frac{a_{2}}{m_{1}^{(n-1)}+k_{2}} \text {. }
$$

From (37) and the expression of $m_{i}^{(n)}$, it immediately follows that

$$
\begin{aligned}
m_{1}^{(n+1)} & =\frac{r_{1}-E q}{b_{1}+\left(a_{1} /\left(m_{2}^{(n)}+k_{1}\right)\right)}-\frac{\varepsilon}{n+1} \\
& >\frac{r_{1}-E q}{b_{1}+\left(a_{1} /\left(m_{2}^{(n-1)}+k_{1}\right)\right)}-\frac{\varepsilon}{n}=m_{1}^{(n)}, \\
m_{2}^{(n+1)} & =\frac{r_{2}}{b_{2}+\left(a_{2} /\left(m_{1}^{(n)}+k_{2}\right)\right)}-\frac{\varepsilon}{n+1} \\
& >\frac{r_{2}}{b_{2}+\left(a_{2} /\left(m_{1}^{(n-1)}+k_{2}\right)\right)}-\frac{\varepsilon}{n}=m_{2}^{(n)} .
\end{aligned}
$$

Therefore,

$$
\begin{array}{ll}
\lim _{t \rightarrow+\infty} M_{1}^{(n)}=\bar{x}, & \lim _{t \rightarrow+\infty} M_{2}^{(n)}=\bar{y}, \\
\lim _{t \rightarrow+\infty} m_{1}^{(n)}=\underline{x}, & \lim _{t \rightarrow+\infty} m_{2}^{(n)}=\underline{y} .
\end{array}
$$

Letting $n \rightarrow+\infty$ in (31), we obtain

$$
\begin{aligned}
& b_{1} \bar{x}+\frac{a_{1} \bar{x}}{\bar{y}+k_{1}}=r_{1}-E q, \\
& b_{2} \bar{y}+\frac{a_{2} \bar{y}}{\bar{x}+k_{2}}=r_{2} ; \\
& b_{1} \underline{x}+\frac{a_{1} \underline{x}}{\underline{y}+k_{1}}=r_{1}-E q, \\
& b_{2} \underline{y}+\frac{a_{2} \underline{y}}{\underline{x}+k_{2}}=r_{2} .
\end{aligned}
$$

(40) shows that $(\bar{x}, \bar{y})$ and $(\underline{x}, \underline{y})$ are positive solutions of the equations

$$
\begin{aligned}
& b_{1} x+\frac{a_{1} x}{y+k_{1}}=r_{1}-E q, \\
& b_{2} y+\frac{a_{2} y}{x+k_{2}}=r_{2} .
\end{aligned}
$$


Wei and Li [1] had already showed that, under the assumption that $r_{1}>E q$ holds, (41) has a unique positive solution $E^{*}\left(x^{*}, y^{*}\right)$. Hence, we conclude that

$$
\bar{x}=\underline{x}=x^{*}, \quad \bar{y}=\underline{y}=y^{*}
$$

that is,

$$
\lim _{t \rightarrow+\infty} x(t)=x^{*}, \quad \lim _{t \rightarrow+\infty} y(t)=y^{*}
$$

Thus, the unique interior equilibrium $E^{*}\left(x^{*}, y^{*}\right)$ is globally attractive. This completes the proof of Theorem 2 .

Proof of Corollary 3. Noting that $M_{1}^{(1)}, M_{2}^{(1)}, m_{1}^{(1)}, m_{2}^{(1)}$ are only dependent on the coefficients of the system (1) and independent of the solution of system (1), hence, (13), (14), (23), and (24) show that the system is permanent. This ends the proof of Corollary 3.

\section{Discussion}

In this paper, we revisited the stability property of a cooperative system incorporating harvesting which was proposed by Wei and Li [1]; by using the iterative method, we show that the condition which ensures the existence of a unique positive equilibrium is enough to ensure the global attractivity of the positive equilibrium. The numeric simulation of Example 1 shows the feasibility of our results. It seems interesting to investigate the stability property of the corresponding discrete type model of system (1); we leave this for future study.

\section{Conflict of Interests}

The authors declare that there is no conflict of interests regarding the publication of this paper.

\section{Acknowledgments}

This work was supported by the Natural Science Foundation of Fujian Province (2013J01011 and 2013J01010) and the Foundation of Fujian Education Bureau (JA13361).

\section{References}

[1] F. Y. Wei and C. Y. Li, "Permanence and globally asymptotic stability of cooperative system incorporating harvesting," Advances in Pure Mathematics, vol. 3, pp. 627-632, 2013.

[2] Y. Li and G. Xu, "Positive periodic solutions for an integrodifferential model of mutualism," Applied Mathematics Letters, vol. 14, no. 5, pp. 525-530, 2001

[3] F. Chen and M. You, "Permanence for an integrodifferential model of mutualism," Applied Mathematics and Computation, vol. 186, no. 1, pp. 30-34, 2007.

[4] F. Chen, X. Liao, and Z. Huang, "The dynamic behavior of $N$ species cooperation system with continuous time delays and feedback controls," Applied Mathematics and Computation, vol. 181, no. 2, pp. 803-815, 2006.
[5] F. Chen, "Permanence of a discrete $N$-species cooperation system with time delays and feedback controls," Applied Mathematics and Computation, vol. 186, no. 1, pp. 23-29, 2007.

[6] F. Chen, "Permanence for the discrete mutualism model with time delays," Mathematical and Computer Modelling, vol. 47, no. 3-4, pp. 431-435, 2008.

[7] F. Chen, J. Yang, L. Chen, and X. Xie, "On a mutualism model with feedback controls," Applied Mathematics and Computation, vol. 214, no. 2, pp. 581-587, 2009.

[8] L. Chen, L. Chen, and Z. Li, "Permanence of a delayed discrete mutualism model with feedback controls," Mathematical and Computer Modelling, vol. 50, no. 7-8, pp. 1083-1089, 2009.

[9] L. Chen and X. Xie, "Permanence of an $N$-species cooperation system with continuous time delays and feedback controls," Nonlinear Analysis: Real World Applications, vol. 12, no. 1, pp. 34-38, 2011.

[10] Y. Li and T. Zhang, "Permanence of a discrete $n$-species cooperation system with time-varying delays and feedback controls," Mathematical and Computer Modelling, vol. 53, no. 56, pp. 1320-1330, 2011.

[11] F. Chen, "On a nonlinear nonautonomous predator-prey model with diffusion and distributed delay," Journal of Computational and Applied Mathematics, vol. 180, no. 1, pp. 33-49, 2005. 


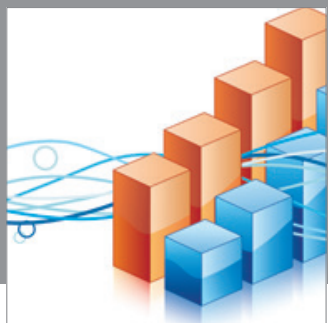

Advances in

Operations Research

mansans

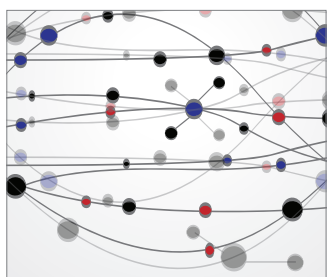

The Scientific World Journal
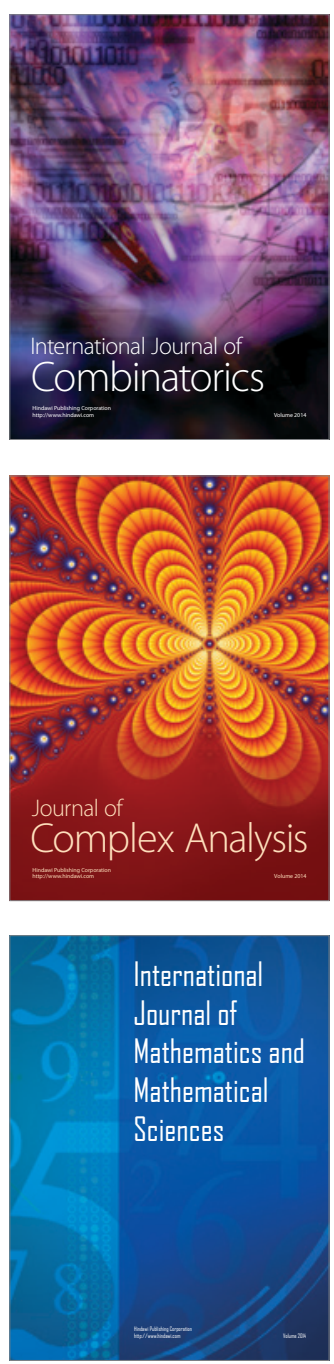
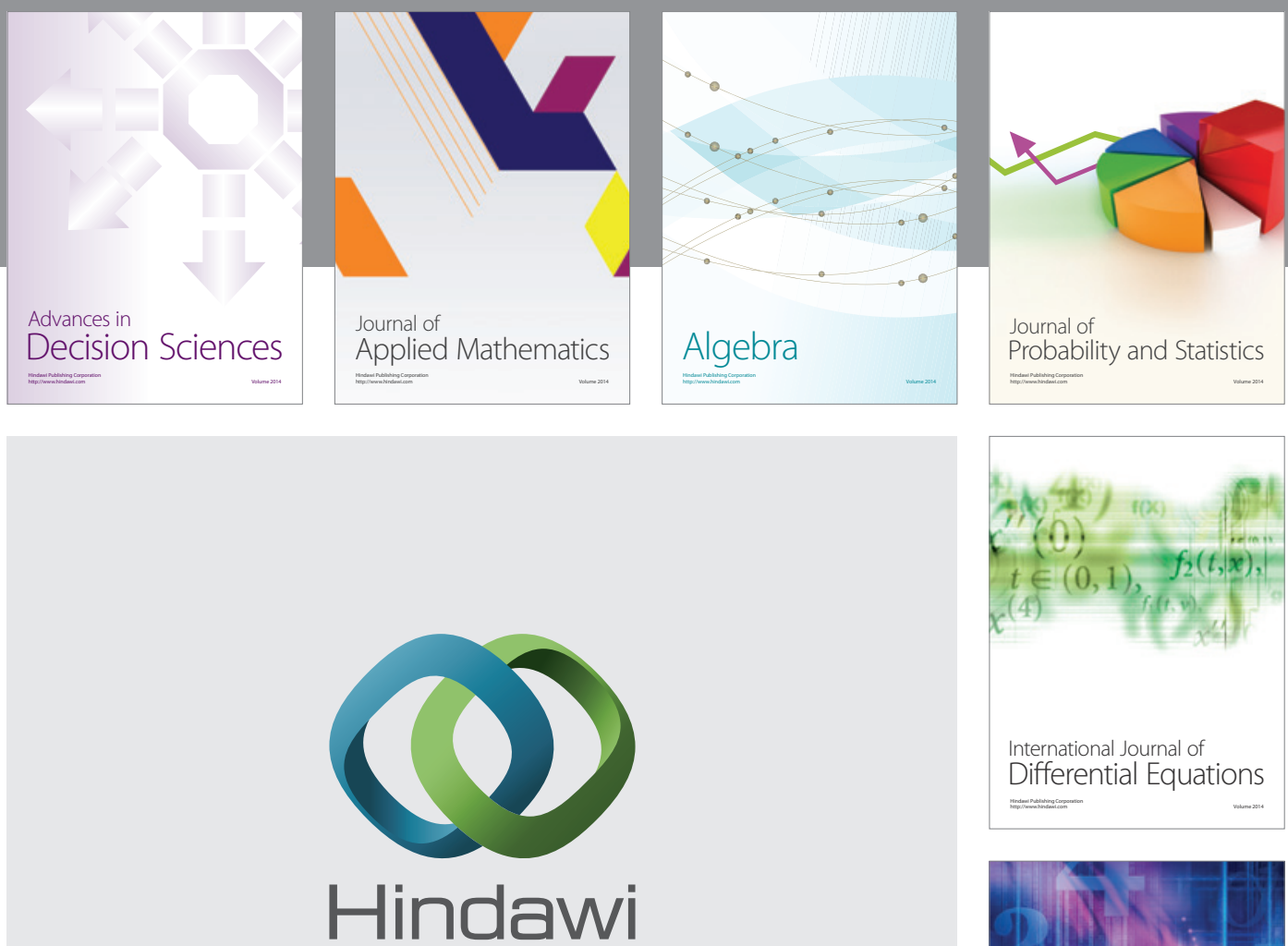

Submit your manuscripts at http://www.hindawi.com
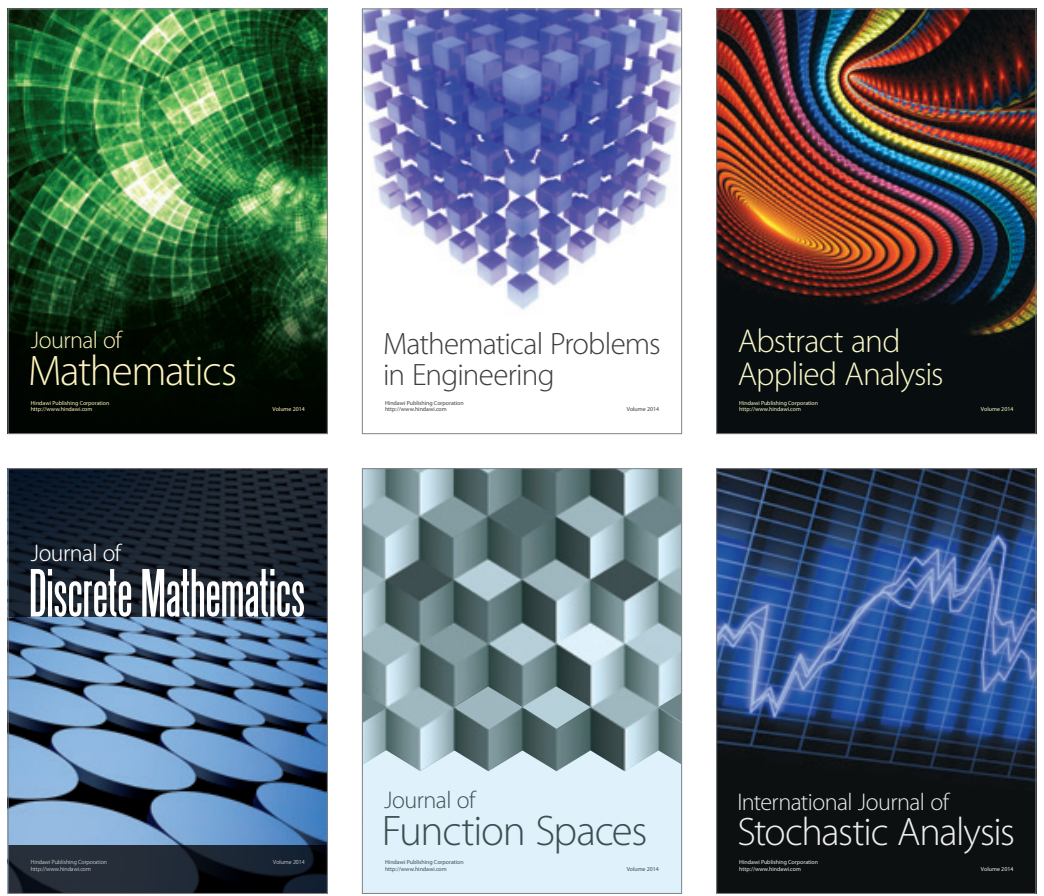

Journal of

Function Spaces

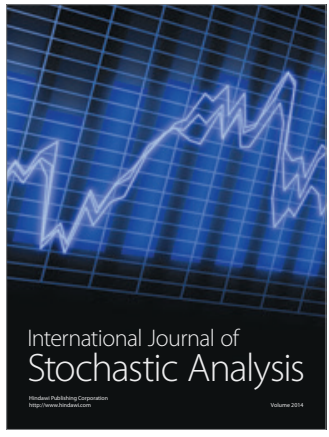

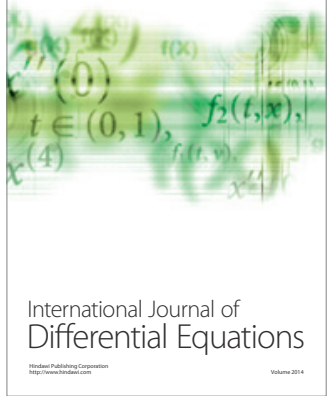
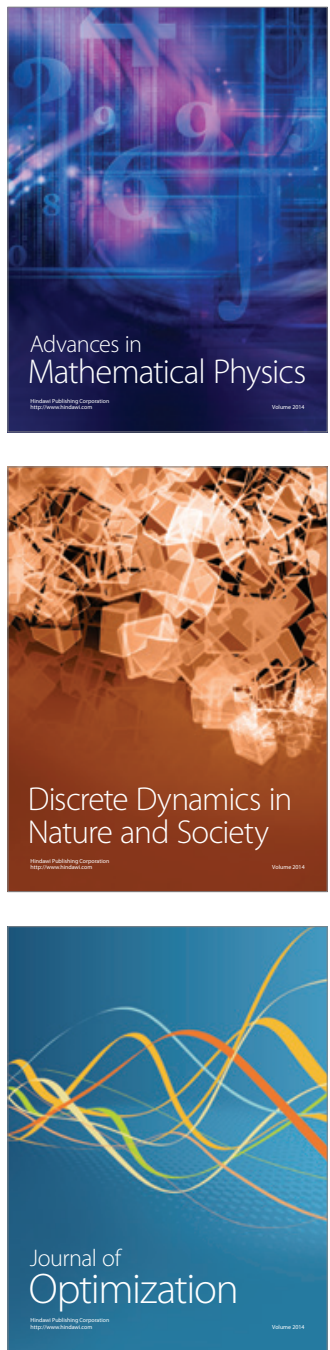